\title{
Ethical clarity in clinical approaches to rabies prevention in re-exposure cases
}

\author{
SAURAV BASU, SUNEELA GARG
}

\begin{abstract}
The lack of adherence to the recommended rabies post-exposure prophylaxis guidelines by physicians during encounters with cases of animal bite re-exposure results in enormous avoidable economic costs, wastage of scarce resources, and is a significant but neglected health concern. The challenges encountered in managing animal bite re-exposure cases arise from deficiencies in the existing health system reflect an overlooked and largely unreported ethical dilemma. Healthcare providers in managing the ethical demands arising from this situation generally tend to uphold the principle of non-maleficence while overlooking the claims of distributive justice and patient autonomy.
\end{abstract}

Keywords: rabies, rabies PEP, animal bite, rabies reexposure, India

Rabies resulting from animal bites is a zoonotic viral disease that is $100 \%$ fatal. It is also completely preventable through timely post-exposure prophylaxis (PEP) with anti-rabies vaccine (ARV) of modern cell-culture origins, effective wound hygiene, and administration of rabies immunoglobulins at the wound- site per protocol (1). However, globally, an estimated 59,000 deaths occur each year, with nearly a third of the burden concentrated in India, due to the large stray dog population in dense urban localities facilitating frequent human-animal encounters (2). The provision of effective PEP for all animal-bite victims is a significant public health challenge in developing countries, due to the high cost of production and supply of ARV and the heavy demand (3). Hence, ensuring continued viable access to ARV, especially without burdening animal-bite patients with out-of-pocket expenses, is primarily a function of the government-funded health system.

Rabies PEP guidelines recommended by the World Health

Authors: Saurav Basu (saurav.basu1983@gmail.com), Senior Resident, Department of Community Medicine, Maulana Azad Medical College, New Delhi, INDIA; Suneela Garg (gargsuneela@gmail.com), Director Professor, Department of Community Medicine, Maulana Azad Medical College, New Delhi, INDIA

To cite: Basu S, Garg S. Ethical clarity in clinical approaches to rabies prevention in re-exposure cases. Indian J Med Ethics. 2021 Jan-Mar; 6(1) NS: 42-43.2020. DOI:10.20529/IJME.2020.091.

Published online first on August 31, 2020

Manuscript Editor:Vijayaprasad Gopichandran

Peer Reviewer: Omesh Kumar Bharti

(c) Indian Journal of Medical Ethics 2020
Organization ( WHO) in 2018 recommend three doses of twosite intradermal (ID) ARV for first-time cases, and two doses of one-site ID ARV with cell culture vaccines for animal bite reexposure cases who had previously received a full course of rabies PEP. An alternative regimen for re-exposed cases includes 4-site ID on day 0.

However, anti-rabies serum (ARS) for category III bites indicating transdermal or mucous membrane exposure is not recommended in re-exposure cases (1). The existing Indian guidelines for rabies PEP recommend four doses of two-site ID ARV in first-time cases (4). This differentiation, reducing the total ARV dose requirement in every re-exposure case with previously completed rabies PEP, is of particular significance in high burden, high prevalence settings like India $(5,6)$. Moreover, studies in India show animal bite re-exposure cases may account for $8.2-28 \%$ of total incident animal bite cases; although large-scale epidemiological evidence is lacking, due to omission in reporting the extent of the phenomenon in existing research $(5,7,8)$.

\section{Overlapping clinical and ethical challenges in animal bite re-exposure cases}

Physicians may often recommend a full course of rabies PEP in animal bite re-exposure cases irrespective of their prior rabies PEP history. The 2020 study conducted by Basu et al, in a secondary care hospital in Delhi, observed that nearly one in every ten animal-bite cases (10.82\%) was receiving extra ARV doses, while, probably, 13 (7.4\%) cases also received unwarranted ARS (6). We have previously discussed that such a practice results in ensuring foolproof protection against rabies, but can potentially cause a significant wastage of ARV and ARS. The situation, when extrapolated to the total annual animal- bite cases, can account for millions of wasted vaccine doses, and the imposition of enormous but avoidable economic and health costs (5). Moreover, the above clinical practice of recommending a full-course of rabies PEP instead of the recommended two-dose single site ID regimen in reexposure cases is, unfortunately, a common occurrence in a developing country like India where patients may frequently lose their health-related documentation.

Under these circumstances, a clinician treating any animal bite case with a history of re-exposure and prior rabies PEP without supportive documentary proof experiences a conflict in negotiating between the ethical principles of non-maleficence and the principle of justice. This is because clinicians cannot 
neglect their patients and expose them to the risk of harm, which justifies their existing conduct in recommending the full course of PEP in all animal bite cases without the documented history of previous PEP. Yet, the likely large-scale wastage of ARV and the opportunity costs imposed on the health system and the patient due to the potentially excessive vaccination also violates the principle of distributive justice. It could also be argued that ignoring the patient's history disregards their treatment choices, thus undermining the principle of patient autonomy in a situation devoid of any overarching public health benefit.

\section{Recommendations}

We recommend the following public health approaches for rationalising the management of animal-bite re-exposure cases in India, to promote adherence to recommended rabies PEP guidelines, prevent excessive use of ARV, and minimise wastage. These strategies will ensure more effective patient protection against rabies while preventing any ethical violations:

\section{Conducting large scale epidemiological studies}

Understanding the actual burden of the problem of excess $A R V$ vaccination is essential. Therefore, epidemiological studies conducted at both community and facility levels would help to identify:

- the proportion of re-exposure cases among incident animal bite cases, and age, gender, socioeconomic status, and region stratification;

- the proportion of re-exposure cases reporting a history of complete rabies PEP with ARV, with and without corroborative medical documentation.

Identifying nationwide practices and self-efficacy of healthcare providers in the management of animal bite reexposure cases

Facility-based studies should ascertain the variety of existing healthcare provider practices and their self- efficacy in managing animal bite re-exposure cases in diverse healthcare settings. Previous studies have reported inadequate awareness of the guidelines for management of animal bite re-exposure cases among Indian physicians $(9,10)$, a problem that is rectifiable through medical curricular updates and training.

\section{Estimating the validity of patient history}

Estimation of previous exposure and management of incident animal-bite cases through corroboration with laboratory estimation of anti-rabies antibody titers is required. Although, such measures may not be cost-effective, they will help generate evidence for the development of practice guidelines.

Similarly, brief training protocols for HCPs are necessary to validate patient history of receipt of ARV by differentiating it from the administration of injection tetanus and any injectable antibiotics.

These measures will help formulate unambiguous and explicit decision-making algorithms for the management of re-exposure cases with a prior history of complete rabies PEP, but without medical documentation. Such steps can then be incorporated into standard treatment guidelines.

\section{Assessment of effectiveness of interventions for preservation and retrieval of medical documentation of animal-bite management}

The effectiveness of interventions to safeguard documentation by HCPs of patients' prior history of ARV needs to be evaluated. Furthermore, research is needed to develop ingenious methods of paper or electronic record keeping for low- resource settings, to facilitate ease of retrieval of previous history of ARV prophylaxis.

\section{Considering newer approaches for rabies management}

The technical feasibility and cost-effectiveness of incorporating the WHO-recommended regimen of 4-sites ID ARV on day 0 for re-exposed cases in Indian health settings, needs to be assessed. If enabled this would halve the number of required health facility visits for re-exposed cases benefiting patients financially and reducing the risk of loss to follow up while also contributing to decongestion at the health settings.

References

1. World Health Organization. Rabies vaccines and immunoglobulins: WHO position paper 2018. Geneva:WHO; 2018 Apr [cited 2020 Mar 9]. Available from: https://www.who.int/immunization/policy/position papers/pp_rabies_summary_2018.pdf?ua $=1$

2. Knobel DL, Cleveland S, Coleman PG, Fevre EM, Melzer MI, Miranda ME et al. Re-evaluating the burden of rabies in Africa and Asia. Bull World Health Organ. 2005 May,83(5):360-8.

3. Hampson K, Cleaveland S, Briggs D. Evaluation of cost-effective strategies for rabies post-exposure vaccination in low-income countries. PLoS Negl Trop Dis. 2011 Mar;5(3):e982.

4. National Health Mission. Guidelines for rabies prophylaxis and intradermal rabies vaccination in Himachal Pradesh, 2019.3rd ed.

5. Basu S, Mariam W, Santra S, Garg S, Singhal R. Re-exposure animal bite management among incident animal bite cases in a secondary care Hospital in Delhi, India. Indian J Public Health 2020;64(1):72-4.

6. Garg S, Basu S, Mahalingam A. Is there a hidden wastage of anti-rabies vaccine and rabies immunoglobulins India due to excess vaccination? Indian Journal of Communicable Diseases. 2016 Jan;2(2):87-9. Doi: 10.21088/ijcd.2395.6631.2216.8

7. Jain P, Jain G. Study of general awareness, attitude, behavior, and practice study on dog bites and its management in the context of prevention of rabies among the victims of dog bite attending the OPD services of CHC Muradnagar. J Fam Med Primary Care. 2014;3(4):355-8.

8. Agarwal AK, Gupta R, Sharma V. A cost evalauation analysis of treatment strategy in re-exposure animal bite cases. Int J Adv Community Med. 2018; 1(1) Part A: 23-6.

9. Garg A, Kumar R, Ingle GK. Knowledge and practices regarding animal bite management and rabies prophylaxis among doctors in Delhi, India. Asia Pac J Public Health. 2013 Jan;25(1):41-7.

10. Chowdhury R, Mukherjee A, Naskar S, Lahiri SK. A study on knowledge of animal bite management and rabies immunization among interns of a government medical college in Kolkata. Int J Med Public Health. $2013 \operatorname{Jan} 13 \cdot 3(1): 17-20$. 